\title{
Energy criteria of global existence for the coupled nonlinear Schrödinger equations with harmonic potentials
}

\author{
Guanggan CHEN \\ College of Mathematics and Software Science, \\ Sichuan Normal University, Chengdu, 610066, China \\ e-mail: chenguanggan@hotmail.com \\ Yunyun WEI \\ College of Information Management, \\ Chengdu University of Technology, Chengdu, 610059, China \\ e-mail: yunyunwei@sina.com
}

Originally submitted as an article authored by Yan Zhang and Guanggan Chen

\begin{abstract}
This paper is concerned with the coupled nonlinear Schrödinger equations with harmonic potentials which describe the Bose-Einstein condensate under the magnetic trap. Two types of the invariant evolution flows are obtained, then a sharp criterion of global existence and blowing up of solutions for the equations is given in terms of the Hamiltonian invariants. Furthermore, we would answer: How small are the initial data such that the global solutions of the system exist?
\end{abstract}

2000 Mathematics Subject Classification: 35Q55, 35B35, 35B60.

Key words: Bose-Einstein condensates; coupled nonlinear Schrödinger equations; global existence.

\section{Introduction}

In this paper we investigate the coupled nonlinear Schrödinger equations with harmonic potentials

$$
\begin{cases}i \varphi_{t}=-\triangle \varphi+|x|^{2} \varphi-|\varphi|^{2} \varphi-|\psi|^{2} \varphi, & \varphi(x, 0)=\varphi_{0} \\ i \psi_{t}=-\triangle \psi+|x|^{2} \psi-|\psi|^{2} \psi-|\varphi|^{2} \psi, & \psi(x, 0)=\psi_{0}\end{cases}
$$


Here $\varphi: \mathbf{R}^{3} \times[0, T) \longrightarrow \mathbf{C}$ and $\psi: \mathbf{R}^{3} \times[0, T) \longrightarrow \mathbf{C}$ are two complex value functions, where $0<T \leq+\infty$. $i=\sqrt{-1}, \triangle$ is the Laplace operator on $\mathbf{R}^{3}$. The coupled equations (1.1) model Bose-Einstein condensates with inter-particle interactions under the magnetic trap $[4,5,19]$. The isotropic harmonic potential function $|x|^{2}$ describes the magnetic field whose role is to confine the movement of particles[4, 5, 19].

From the view point of physics, the following problems are very important and significant. Under what conditions, will the condensates become unstable to collapse (blowup)? And under what conditions, will the condensates exist for all time (global existence)? Especially the sharp criteria of blowing up and global existence are pursued strongly(also see [7, 8, 9, 23, 25, 26]). From the view point of mathematics, the problems are rather fascinating and challenging. Strauss[21] and Cazenave[6] also mentioned these topics in their monographs.

In the following we firstly recall the classical nonlinear Schrödinger equation

$$
i \varphi_{t}=-\triangle \varphi-|\varphi|^{p-1} \varphi, \quad \varphi \in H^{1}\left(\mathbf{R}^{D}\right),
$$

where $1 \leq p<\frac{D+2}{D-2}$ when $D \geq 3$ and $1 \leq p<\infty$ when $D=1,2$. Ginibre and Velo $[11,12]$ established the local well-posedness of the Cauchy problem (1.2) in energy space $H^{1}\left(\mathbf{R}^{D}\right)$. Furthermore they also got the global existence for both small initial data and all initial data with $1 \leq p<1+\frac{4}{D}$. When $p \geq 1+\frac{4}{D}$, Glassey [10], Ogawa and Tsutsumi $[16,17]$ showed that the solutions of the Cauchy problem (1.2) blow up in a finite time for large initial data, especially for negative energy initial data. Thus $p=1+\frac{4}{D}$ is called the critical nonlinear power exponent and $p>1+\frac{4}{D}$ is called the supercritical nonlinear power exponent. A nature question appears for $p \geq 1+\frac{4}{D}$. Can one find some sharp criteria for global existence and blowing up of the solutions corresponding to the initial data? Weinstein [24] gave a crucial criterion in terms of $L^{2}$-mass initial data. Berestycki and Cazenave [2], Berestycki, Gallouét and Kavian [3], Kuznetsov et al.[13], Zhang [25] gave some sharp criteria in terms of energy initial data.

In this paper, for system (1.1), firstly, we define the energy space as

$$
\Sigma:=\left\{u \in H^{1}\left(\mathbf{R}^{3}\right): \int|x|^{2}|u|^{2} d x<\infty\right\} .
$$

Here and hereafter, for simplicity, we denote $\int_{\mathbf{R}^{3}} \cdot d x$ by $\int \cdot d x$. $\Sigma$ becomes a Hilbert space, continuously embedded in $H^{1}\left(\mathbf{R}^{3}\right)$, when endowed with the inner product

$$
\langle\varphi, \phi\rangle_{\Sigma}=\int\left[\nabla \varphi \nabla \bar{\phi}+\varphi \bar{\phi}+|x|^{2} \varphi \bar{\phi}\right] d x,
$$

whose associated norm we denote by $\|\cdot\|_{\Sigma}$. In addition, we use $\|\cdot\|_{2}$ to denote the norm of $L^{2}\left(\mathbf{R}^{3}\right)$. From Cazenave[6] and $\mathrm{Oh}[15]$, the local well-posedness result of system (1.1) is as follows.

Assume $\left(\varphi_{0}, \psi_{0}\right) \in \Sigma \times \Sigma$. Then there exists a unique pair of solutions $(\varphi, \psi)$ of the Cauchy problem $(1.1)$ in $C([0, T) ; \Sigma \times \Sigma)$ for some $T \in[0, \infty)$ (maximal 
existence time). Furthermore, we have the following alternatives: $T=\infty$ (global existence) or else $T<\infty$ and $\lim _{t \rightarrow T}\left(\|\varphi\|_{\Sigma}+\|\psi\|_{\Sigma}\right)=\infty$ (blowing up).

In this paper, for system (1.1), we are also interested in the problem of searching a sharp criteria of blowing up and global existence.

For equations (1.1), it is deserved to noted that they are heavily different from the classic nonlinear Schrödinger equation (1.2)(see Zhang[26]), which is one of the motivations for us to consider equations (1.1). The essential reason is that equations (1.1) possess the harmonic potentials $|x|^{2} \varphi$ and $|x|^{2} \psi$, which not only cause to lose the classic scaling invariance, while it exists in equation (1.2), but also cause to appear much difficulty in solving the global existence and in proving the blowing up. We exploit the Hamiltonian invariants of equations (1.1) and apply a series of sharp interpolations equalities to get a sharp criterion for equations (1.1). The principal results reads as follows.

Theorem 1.1 Let $Q(x)$ be the positive and spherically symmetric ground state solution of the field equation

$$
-\triangle u+u-|u|^{2} u=0, \quad u \in H^{1}\left(\mathbf{R}^{3}\right) .
$$

(Here the existence of $Q$ see Strauss [20] and the uniqueness of $Q$ see Kwong [14].) Denote $(\varphi, \psi)$ the solutions of the Cauchy problem (1.1) corresponding to the initial data $\left(\varphi_{0}, \psi_{0}\right)$. When the initial data $\left(\varphi_{0}, \psi_{0}\right) \in \Sigma \times \Sigma$ satisfy

$$
\begin{aligned}
L\left(\varphi_{0}, \psi_{0}\right):= & \left.\int|| \nabla \varphi_{0}\right|^{2}+\left|\nabla \psi_{0}\right|^{2}+|x|^{2}\left|\varphi_{0}\right|^{2}+|x|^{2}\left|\psi_{0}\right|^{2}+\left|\varphi_{0}\right|^{2}+\left|\psi_{0}\right|^{2} \\
& \left.-\left|\varphi_{0}\right|^{2}\left|\psi_{0}\right|^{2}-\frac{1}{2}\left|\varphi_{0}\right|^{4}-\frac{1}{2}\left|\psi_{0}\right|^{4}\right] d x \\
< & \frac{\sqrt{2}}{4}\|Q\|_{2}^{2},
\end{aligned}
$$

one has

1. if $\Theta\left(\varphi_{0}, \psi_{0}\right):=\int\left[\left|\nabla \varphi_{0}\right|^{2}+\left|\nabla \psi_{0}\right|^{2}+|x|^{2}\left|\varphi_{0}\right|^{2}+|x|^{2}\left|\psi_{0}\right|^{2}\right] d x<\frac{3 \sqrt{2}}{4}\|Q\|_{2}^{2}$, then $(\varphi, \psi)$ globally exist in $\Sigma \times \Sigma$;

2. if $\Theta\left(\varphi_{0}, \psi_{0}\right):=\int\left[\left|\nabla \varphi_{0}\right|^{2}+\left|\nabla \psi_{0}\right|^{2}+|x|^{2}\left|\varphi_{0}\right|^{2}+|x|^{2}\left|\psi_{0}\right|^{2}\right] d x>\frac{3 \sqrt{2}}{4}\|Q\|_{2}^{2}$, then $(\varphi, \psi)$ blow up in a finite time.

Remark 1.1 Theorem 1.1 indicates that the ground state solution $Q$ of the stationary state equation (1.5) affects strongly the solutions of the evolution equation (1.1).

Remark 1.2 We pose a set

$$
\left\{\left(\varphi_{0}, \psi_{0}\right) \in \Sigma \times \Sigma: \quad \Theta\left(\varphi_{0}, \psi_{0}\right)<\frac{3 \sqrt{2}}{4}\|Q\|_{2}^{2} \quad \text { and } \quad L\left(\varphi_{0}, \psi_{0}\right)<\frac{\sqrt{2}}{4}\|Q\|_{2}^{2}\right\} .
$$


It is deserved to note that the set is a unbounded set with respect to the $L^{2}$-mass $\left\|\varphi_{0}\right\|_{2}$ and $\left\|\psi_{0}\right\|_{2}$. Therefore, Theorem 1.1 gives an interesting result for global existence for which we can take initial values with the $L^{2}$-mass $\left\|\varphi_{0}\right\|_{2}$ and $\left\|\psi_{0}\right\|_{2}$ large as we want.

In addition, one can also answer the question: how small are the initial data in $\Sigma \times \Sigma$ such that the solutions of the system globally exist.

Theorem 1.2 Let $\left(\varphi_{0}, \psi_{0}\right) \in \Sigma \times \Sigma$ satisfy

$$
\begin{aligned}
\left\|\varphi_{0}\right\|_{\Sigma}^{2}+\left\|\psi_{0}\right\|_{\Sigma}^{2} & =\int\left[\left|\nabla \varphi_{0}\right|^{2}+\left|\nabla \psi_{0}\right|^{2}+|x|^{2}\left|\varphi_{0}\right|^{2}+|x|^{2}\left|\psi_{0}\right|^{2}+\left|\varphi_{0}\right|^{2}+\left|\psi_{0}\right|^{2}\right] d x \\
& <\frac{\sqrt{2}}{4}\|Q\|_{2}^{2}
\end{aligned}
$$

Then the solutions $(\varphi, \psi)$ of the Cauchy problem (1.1) globally exist in $\Sigma \times \Sigma$.

This paper is organized as follows. In the following, we first state some preliminaries concerned. Then we establish the invariant evolution flows of equations (1.1). At last, we prove Theorem 1.1 and Theorem 1.2.

\section{$2 \quad$ Preliminaries}

Firstly, we give the conservation laws of equations (1.1) as follows.

Proposition 2.1 Let $\left(\varphi_{0}, \psi_{0}\right) \in \Sigma \times \Sigma$ and $(\varphi, \psi)$ be the solutions of the Cauchy problem (1.1) on $t \in[0, T)$. Then for all $t \in[0, T),(\varphi, \psi)$ satisfy the following two conservation laws of the mass

$$
\begin{aligned}
& M(\varphi(t)):=\frac{1}{2} \int|\varphi(t)|^{2} d x=M\left(\varphi_{0}\right), \\
& M(\psi(t)):=\frac{1}{2} \int|\psi(t)|^{2} d x=M\left(\psi_{0}\right),
\end{aligned}
$$

and energy

$$
\begin{aligned}
E(\varphi(t), \psi(t)):= & \frac{1}{2} \int\left[|\nabla \varphi(t)|^{2}+|\nabla \psi(t)|^{2}+|x|^{2}|\varphi(t)|^{2}+|x|^{2}|\psi(t)|^{2}\right. \\
& \left.-|\varphi(t)|^{2}|\psi(t)|^{2}-\frac{1}{2}|\varphi(t)|^{4}-\frac{1}{2}|\psi(t)|^{4}\right] d x \\
= & E\left(\varphi_{0}, \psi_{0}\right) .
\end{aligned}
$$

Proof. We use a formal argument of Ozawa[18] to prove it as follows (also see cazenave[6]). 
Using $\bar{\varphi}$ (the conjugation of $\varphi$ ) to multiply the first equation of (1.1) and integrating on $\mathbf{R}^{3}$, then taking the imaginary part, we easily obtain $\int|\varphi(t)|^{2} d x$ $=\int\left|\varphi_{0}\right|^{2} d x$. At the same time, using $\bar{\psi}$ to multiply the second equation of (1.1) and integrating on $\mathbf{R}^{3}$, then taking the imaginary part, we also easily obtain $\int|\psi(t)|^{2} d x=\int\left|\psi_{0}\right|^{2} d x$.

Using $\bar{\varphi}_{t}$ to multiply the first equation of (1.1) and integrating on $\mathbf{R}^{3}$, then taking the real part, we obtain

$$
\int\left[\frac{d}{d t}|\nabla \varphi|^{2}+|x|^{2} \frac{d}{d t}|\varphi|^{2}-|\varphi|^{2} \frac{d}{d t}|\varphi|^{2}-|\psi|^{2} \frac{d}{d t}|\varphi|^{2}\right] d x=0
$$

At the same time, using $\bar{\psi}_{t}$ to multiply the second equation of (1.1) and integrating on $\mathbf{R}^{3}$, then taking the real part, we also obtain

$$
\int\left[\frac{d}{d t}|\nabla \psi|^{2}+|x|^{2} \frac{d}{d t}|\psi|^{2}-|\psi|^{2} \frac{d}{d t}|\psi|^{2}-|\varphi|^{2} \frac{d}{d t}|\psi|^{2}\right] d x=0
$$

Then it follows from $(2.3)$ and $(2.4)$ that $E(\varphi, \psi)=E\left(\varphi_{0}, \psi_{0}\right)$

Proposition 2.2 Let $\left(\varphi_{0}, \psi_{0}\right) \in \Sigma \times \Sigma$ and let $(\varphi, \psi)$ be the solutions of the Cauchy problem (1.1) on $[0, T)$. Put $J(t)=\int|x|^{2}\left(\left.\varphi\right|^{2}+|\psi|^{2}\right) d x$. Then one has

$$
\begin{aligned}
\frac{d^{2}}{d t^{2}} J(t)= & 8 \int\left[|\nabla \varphi|^{2}+|\nabla \psi|^{2}-|x|^{2}|\varphi|^{2}-|x|^{2}|\psi|^{2}-\frac{3}{2}|\varphi|^{2}|\psi|^{2}\right. \\
& \left.-\frac{3}{4}|\varphi|^{4}-\frac{3}{4}|\psi|^{4}\right] d x
\end{aligned}
$$

Proof. We use a formal argument of Cazenave[6] to prove it.

Since $(\varphi, \psi) \in C([0, T), \Sigma \times \Sigma)$ are the solutions of the Cauchy problem (1.1), we can obtain by directly calculating that

$$
\frac{d}{d t} J(t)=-4 \operatorname{Im} \int x \varphi \nabla \bar{\varphi} d x-4 \operatorname{Im} \int x \psi \nabla \bar{\psi} d x
$$

On the other hand, we have

$$
\begin{aligned}
& \int x \varphi \nabla \bar{\varphi}_{t} d x=-\int \bar{\varphi}_{t} \nabla(x \varphi) d x=-\int \bar{\varphi}_{t}(3 \varphi+x \nabla \varphi) d x \\
& \int x \psi \nabla \bar{\psi}_{t} d x=-\int \bar{\psi}_{t} \nabla(x \psi) d x=-\int \bar{\psi}_{t}(3 \psi+x \nabla \psi) d x .
\end{aligned}
$$


Therefore

$$
\begin{aligned}
\frac{d^{2}}{d t^{2}} J(t)= & -4 \operatorname{Im} \int x\left(\varphi_{t} \nabla \bar{\varphi}+\varphi \nabla \bar{\varphi}_{t}\right) d x-4 \operatorname{Im} \int x\left(\psi_{t} \nabla \bar{\psi}+\psi \nabla \bar{\psi}_{t}\right) d x \\
= & 8 \operatorname{Re} \int x \nabla \bar{\varphi}\left(\triangle \varphi-|x|^{2} \varphi+|\varphi|^{2} \varphi+|\psi|^{2} \varphi\right) d x \\
& -4 \operatorname{Re} \int 3 \varphi\left(\triangle \bar{\varphi}-|x|^{2} \bar{\varphi}+|\varphi|^{2} \bar{\varphi}+|\psi|^{2} \bar{\varphi}\right) d x \\
& +8 \operatorname{Re} \int x \nabla \bar{\psi}\left(\triangle \psi-|x|^{2} \psi+|\psi|^{2} \psi+|\varphi|^{2} \psi\right) d x \\
& -4 \operatorname{Re} \int 3 \psi\left(\triangle \bar{\psi}-|x|^{2} \bar{\psi}+|\psi|^{2} \bar{\psi}+|\varphi|^{2} \bar{\psi}\right) d x .
\end{aligned}
$$

At the same time, we have

$$
\begin{aligned}
& \operatorname{Re} \int x(\Delta \varphi) \nabla \bar{\varphi} d x=\frac{1}{2} \int|\nabla \varphi|^{2} d x, \\
& \operatorname{Re} \int x(\Delta \psi) \nabla \bar{\psi} d x=\frac{1}{2} \int|\nabla \psi|^{2} d x, \\
& \operatorname{Re} \int \varphi \Delta \bar{\varphi} d x=-\int|\nabla \varphi|^{2} d x, \\
& \operatorname{Re} \int \psi \triangle \bar{\psi} d x=-\int|\nabla \psi|^{2} d x \\
& \operatorname{Re} \int x \nabla \bar{\varphi}\left(|x|^{2} \varphi\right) d x=-\frac{5}{2} \int|x|^{2}|\varphi|^{2} d x, \\
& \operatorname{Re} \int x \nabla \bar{\psi}\left(|x|^{2} \psi\right) d x=-\frac{5}{2} \int|x|^{2}|\psi|^{2} d x, \\
& \operatorname{Re} \int x \nabla \bar{\varphi}\left(-|\varphi|^{2} \varphi\right) d x=\frac{3}{4} \int|\varphi|^{4} d x, \\
& \operatorname{Re} \int x \nabla \bar{\psi}\left(-|\psi|^{2} \psi\right) d x=\frac{3}{4} \int|\psi|^{4} d x .
\end{aligned}
$$

Note that

$\operatorname{Re} \int x \nabla \bar{\varphi}\left(-|\psi|^{2} \varphi\right) d x+\operatorname{Re} \int x \nabla \bar{\psi}\left(-|\varphi|^{2} \psi\right) d x=-\frac{3}{2} \int|\varphi|^{2}|\psi|^{2} d x$. true.

Therefore it follows immediately from (2.8), (2.9) and (2.10) that (2.5) is

Lemma 2.1 ([1]) Let $Q(x)$ be the positive and spherically symmetric ground state solution of the field equation (1.5)

$$
-\triangle u+u-|u|^{2} u=0, \quad u \in H^{1}\left(\mathbf{R}^{3}\right) .
$$


Then the best constant $C_{*}>0$ of the Gagliardo-Nirenberg's inequality,

$$
\|f\|_{4}^{4} \leq C_{*}\|f\|_{2}\|\nabla f\|_{2}^{3},
$$

is given by

$$
C_{*}=\frac{4}{3 \sqrt{3}\|Q\|_{2}^{2}} .
$$

Lemma 2.2 ([22] Young inequality) For any positive real number $a>0, b$ $>0, \varepsilon>0$, let $r$ satisfy $r \in(1,+\infty)$ and $r^{\prime}$ satisfy $1 / r+1 / r^{\prime}=1$.

Then

$$
a b \leq \frac{\varepsilon}{r} a^{r}+\frac{1}{r^{\prime} \varepsilon^{r^{\prime} / r}} b^{r^{\prime}} .
$$

Lemma 2.3 ([24]) Let $\varphi \in \Sigma$. Then one has

$$
\int|\varphi|^{2} d x \leq \frac{2}{3}\left(\int|\nabla \varphi|^{2} d x\right)^{1 / 2}\left(\int|x|^{2}|\varphi|^{2} d x\right)^{1 / 2}
$$

\section{Invariant evolution flows}

Firstly, we pose

$$
\begin{aligned}
& K_{g}:=\left\{(u, v): \Theta(u, v)<\frac{3 \sqrt{2}}{4}\|Q\|_{2}^{2} \quad \text { and } \quad L(u, v)<\frac{\sqrt{2}}{4}\|Q\|_{2}^{2}\right\}, \\
& K_{b}:=\left\{(u, v): \Theta(u, v)>\frac{3 \sqrt{2}}{4}\|Q\|_{2}^{2} \quad \text { and } \quad L(u, v)<\frac{\sqrt{2}}{4}\|Q\|_{2}^{2}\right\},
\end{aligned}
$$

where $L$ and $\Theta$ are defined as in Theorem 1.1, and $Q$ is the ground state solution of nonlinear elliptic equation (1.5).

So we have local invariant flows theorem.

Theorem 3.1 $K_{g}$ and $K_{b}$ are invariant under the flow generated by the Cauchy problem (1.1). More precisely, if the initial data $\left(\varphi_{0}, \psi_{0}\right) \in K_{g}\left(K_{b}\right)$, then the solutions $(\varphi, \psi)$ still satisfy $(\varphi, \psi) \in K_{g}\left(K_{b}\right)$.

Proof. Let $\left(\varphi_{0}, \psi_{0}\right) \in K_{g}$ and let $(\varphi(t), \psi(t))$ be the solutions of the Cauchy problem (1.1) with the initial data $\left(\varphi_{0}, \psi_{0}\right)$. By Proposition 2.1, one has $L(\varphi(t), \psi(t))=2[E(\varphi(t), \psi(t))+M(\varphi(t))+M(\psi(t))]=2\left[E\left(\varphi_{0}, \psi_{0}\right)+M\left(\varphi_{0}\right)\right.$ $\left.+M\left(\psi_{0}\right)\right]=L\left(\varphi_{0}, \psi_{0}\right), t \in[0, T)$. Thus from $L\left(\varphi_{0}, \psi_{0}\right)<\frac{\sqrt{2}}{4}\|Q\|_{2}^{2}$, it follows that

$$
L(\varphi(t), \psi(t))<\frac{\sqrt{2}}{4}\|Q\|_{2}^{2}, \quad t \in[0, T) .
$$


To check $(\varphi(t), \psi(t)) \in K_{g}$, we need to prove that

$$
\Theta(\varphi(t), \psi(t))<\frac{3 \sqrt{2}}{4}\|Q\|_{2}^{2}, \quad t \in[0, T) .
$$

If (3.2) were not true, by continuity, because of $\Theta\left(\varphi_{0}, \psi_{0}\right)<\frac{3 \sqrt{2}}{4}\|Q\|_{2}^{2}$, there would exist a $t_{1} \in[0, T)$ such that

$$
\Theta\left(\varphi\left(t_{1}\right), \psi\left(t_{1}\right)\right)=\frac{3 \sqrt{2}}{4}\|Q\|_{2}^{2}
$$

However, it follows from Lemma 2.1 that

$$
\begin{aligned}
L\left(\varphi\left(t_{1}\right), \psi\left(t_{1}\right)\right)= & \int\left[\left|\nabla \varphi\left(t_{1}\right)\right|^{2}+\left|\nabla \psi\left(t_{1}\right)\right|^{2}+|x|^{2}\left|\varphi\left(t_{1}\right)\right|^{2}+|x|^{2}\left|\psi\left(t_{1}\right)\right|^{2}\left|\varphi\left(t_{1}\right)\right|^{2}\right. \\
& \left.+\left|\psi\left(t_{1}\right)\right|^{2}-\left|\varphi\left(t_{1}\right)\right|^{2}\left|\psi\left(t_{1}\right)\right|^{2}-\frac{1}{2}\left|\varphi\left(t_{1}\right)\right|^{4}-\frac{1}{2}\left|\psi\left(t_{1}\right)\right|^{4}\right] d x \\
\geq & \int\left[\left|\nabla \varphi\left(t_{1}\right)\right|^{2}+\left|\nabla \psi\left(t_{1}\right)\right|^{2}+|x|^{2}\left|\varphi\left(t_{1}\right)\right|^{2}+|x|^{2}\left|\psi\left(t_{1}\right)\right|^{2}\right. \\
& \left.+\left|\varphi\left(t_{1}\right)\right|^{2}+\left|\psi\left(t_{1}\right)\right|^{2}-\left|\varphi\left(t_{1}\right)\right|^{4}-\left|\psi\left(t_{1}\right)\right|^{4}\right] d x \\
\geq & \int\left[\left|\nabla \varphi\left(t_{1}\right)\right|^{2}+\left|\nabla \psi\left(t_{1}\right)\right|^{2}+|x|^{2}\left|\varphi\left(t_{1}\right)\right|^{2}+|x|^{2}\left|\psi\left(t_{1}\right)\right|^{2}\right] d x \\
& +\left\|\varphi\left(t_{1}\right)\right\|_{2}^{2}+\left\|\psi\left(t_{1}\right)\right\|_{2}^{2}-C_{*}\left\|\varphi\left(t_{1}\right)\right\|_{2}\left\|\varphi\left(t_{1}\right)\right\|_{2}^{3} \\
& -C_{*}\left\|\psi\left(t_{1}\right)\right\|_{2}\left\|\psi\left(t_{1}\right)\right\|_{2}^{3} .
\end{aligned}
$$

Here $C_{*}$ is the best constant of Gagliardo-Nirenberg's inequality as in Lemma 2.1. Using the Young inequality (Lemma 2.2), we have

$$
\left\|\varphi\left(t_{1}\right)\right\|_{2}\left\|\nabla \varphi\left(t_{1}\right)\right\|_{2}^{3} \leq \frac{\varepsilon}{2}\left[\left\|\varphi\left(t_{1}\right)\right\|_{2}^{2}+\frac{1}{2 \varepsilon}\left[\left\|\nabla \varphi\left(t_{1}\right)\right\|_{2}^{6}\right.\right.
$$

and

$$
\left\|\psi\left(t_{1}\right)\right\|_{2}\left\|\nabla \psi\left(t_{1}\right)\right\|_{2}^{3} \leq \frac{\varepsilon}{2}\left[\left\|\psi\left(t_{1}\right)\right\|_{2}^{2}+\frac{1}{2 \varepsilon}\left[\left\|\nabla \psi\left(t_{1}\right)\right\|_{2}^{6},\right.\right.
$$

where $\varepsilon>0$ is a arbitrary positive constant. Choose $\varepsilon>0$ satisfying $\frac{\varepsilon}{2} C_{*}=1$, i.e.,

$$
\varepsilon=\frac{2}{C_{*}} .
$$


Then

$$
\begin{aligned}
L\left(\varphi\left(t_{1}\right), \psi\left(t_{1}\right)\right) \geq & \int\left[\left|\nabla \varphi\left(t_{1}\right)\right|^{2}+\left|\nabla \psi\left(t_{1}\right)\right|^{2}+|x|^{2}\left|\varphi\left(t_{1}\right)\right|^{2}+|x|^{2}\left|\psi\left(t_{1}\right)\right|^{2}\right] d x \\
& -\frac{C_{*}^{2}}{4}\left(\int\left[\left|\nabla \varphi\left(t_{1}\right)\right|^{2}+|x|^{2}\left|\varphi\left(t_{1}\right)\right|^{2}\right] d x\right)^{3} \\
& -\frac{C_{*}^{2}}{4}\left(\int\left[\left|\nabla \psi\left(t_{1}\right)\right|^{2}+|x|^{2}\left|\psi\left(t_{1}\right)\right|^{2}\right] d x\right)^{3} \\
\geq & \int\left[\left|\nabla \varphi\left(t_{1}\right)\right|^{2}+\left|\nabla \psi\left(t_{1}\right)\right|^{2}+|x|^{2}\left|\varphi\left(t_{1}\right)\right|^{2}+|x|^{2}\left|\psi\left(t_{1}\right)\right|^{2}\right] d x \\
& -\frac{C_{*}^{2}}{2}\left(\int \left[\left|\nabla \varphi\left(t_{1}\right)\right|^{2}+\left|\nabla \psi\left(t_{1}\right)\right|^{2}+|x|^{2}\left|\varphi\left(t_{1}\right)\right|^{2}\right.\right. \\
& \left.\left.+|x|^{2}\left|\psi\left(t_{1}\right)\right|^{2}\right] d x\right)^{3} .
\end{aligned}
$$

Set a real value function

$$
F(\Theta):=\Theta-\frac{C_{*}^{2}}{2} \Theta^{3} \quad \text { for any } \quad \Theta>0 .
$$

For $F(\Theta)$ on $\Theta \in(0,+\infty)$, one has

1. when $\Theta=\sqrt{\frac{2}{3}} \frac{1}{C_{*}}>0$, then $F^{\prime}(\Theta)=0$;

2. for any $\Theta \in(0,+\infty)$, then $F^{\prime \prime}(\Theta)<0$.

Therefore, $F(\Theta)$ reaches the maximum

$$
F_{\max }=\frac{2}{3} \sqrt{\frac{2}{3}} \frac{1}{C_{*}}=\frac{\sqrt{2}}{2}\|Q\|_{2}^{2}, \quad \text { at } \quad \Theta=\sqrt{\frac{2}{3}} \frac{1}{C_{*}}=\frac{3 \sqrt{2}}{4}\|Q\|_{2}^{2} .
$$

Obviously, $F_{\max }=\frac{\sqrt{2}}{2}\|Q\|_{2}^{2}>\frac{\sqrt{2}}{4}\|Q\|_{2}^{2}$. Therefore, it follows from (3.3), (3.8) and (3.9) that

$$
L\left(\varphi\left(t_{1}\right), \psi\left(t_{1}\right)\right)>\frac{\sqrt{2}}{4}\|Q\|_{2}^{2},
$$

which violates $L\left(\varphi\left(t_{1}\right), \psi\left(t_{1}\right)\right)=L\left(\varphi_{0}, \psi_{0}\right)<\frac{\sqrt{2}}{4}\|Q\|_{2}^{2}$. Then (3.2) is true. Hence, $K_{g}$ is invariant under the flow generated by the Cauchy problem (1.1).

By the same argument as the above, we can show that $K_{b}$ is also invariant under the flow generated by the Cauchy problem (1.1).

\section{Sharp criterion}

In this section, we shall prove the main results Theorem 1.1 and Theorem 1.2. 


\section{Proof of Theorem 1.1}

1. Let $\left(\varphi_{0}, \psi_{0}\right) \in K_{g}$ and let $(\varphi, \psi)$ be the solutions of the Cauchy problem (1.1) with the initial data $\left(\varphi_{0}, \psi_{0}\right)$ in $t \in[0, T)$. It follows from Theorem 3.1 that $(\varphi, \psi) \in K_{g}$. Hence

$$
\Theta(\varphi, \psi)=\int\left[|\nabla \varphi|^{2}+|\nabla \psi|^{2}+|x|^{2}|\varphi|^{2}+|x|^{2}|\psi|^{2}\right] d x<\frac{3 \sqrt{2}}{4}\|Q\|_{2}^{2} .
$$

At the same time, noting $\int|\varphi|^{2} d x=\int\left|\varphi_{0}\right|^{2} d x$ and $\int|\psi|^{2} d x=\int\left|\psi_{0}\right|^{2} d x$ from (2.1), then we easily obtain $(\varphi, \psi)$ are bounded in $\Sigma \times \Sigma$. Therefore, from the local well-posedness result, the solution $(\varphi, \psi)$ of the Cauchy problem (1.1) globally exist in $\Sigma \times \Sigma$.

2. Let $\left(\varphi_{0}, \psi_{0}\right) \in K_{b}$ and let $(\varphi, \psi)$ be the solutions of the Cauchy problem (1.1) with the initial data $\left(\varphi_{0}, \psi_{0}\right)$ in $t \in[0, T)$. It follows from Theorem 3.1 that $(\varphi, \psi) \in K_{b}$, which reads

$$
\Theta(\varphi, \psi)=\int\left[|\nabla \varphi|^{2}+|\nabla \psi|^{2}+|x|^{2}|\varphi|^{2}+|x|^{2}|\psi|^{2}\right] d x>\frac{3 \sqrt{2}}{4}\|Q\|_{2}^{2},
$$

and

$$
\begin{aligned}
L(\varphi, \psi)= & \int\left[|\nabla \varphi|^{2}+|\nabla \psi|^{2}+|x|^{2}|\varphi|^{2}+|x|^{2}|\psi|^{2}\right. \\
& \left.+|\varphi|^{2}+|\psi|^{2}-|\varphi|^{2}|\psi|^{2}-\frac{1}{2}|\varphi|^{4}-\frac{1}{2}|\psi|^{4}\right] d x \\
< & \frac{\sqrt{2}}{4}\|Q\|_{2}^{2} .
\end{aligned}
$$

Therefore it follows from Proposition 2.2, Proposition 2.1, (4.2) and (4.3) that

$$
\begin{aligned}
\frac{d^{2}}{d t^{2}} \int & |x|^{2}|\varphi|^{2} d x=8 \int\left[|\nabla \varphi|^{2}+|\nabla \psi|^{2}-|x|^{2}|\varphi|^{2}-|x|^{2}|\psi|^{2}\right. \\
& \left.-\frac{3}{2}|\varphi|^{2}|\psi|^{2}-\frac{3}{4}|\varphi|^{4}-\frac{3}{4}|\psi|^{4}\right] d x \\
= & 8\left[L(\varphi, \psi)-\frac{1}{2} \int\left[|\varphi|^{2}|\psi|^{2}+\frac{1}{2}|\varphi|^{4}+\frac{1}{2}|\psi|^{4}\right] d x\right] \\
& -16 \int\left[|x|^{2}|\varphi|^{2}+|x|^{2}|\psi|^{2}\right] d x-8 \int\left[|\varphi|^{2}+|\psi|^{2}\right] d x \\
= & 8\left[L(\varphi, \psi)-\frac{1}{2}\left[\int\left(|\nabla \varphi|^{2}+|\nabla \psi|^{2}+|x|^{2}|\varphi|^{2}+|x|^{2}|\psi|^{2}\right) d x\right.\right. \\
& -L(\varphi, \psi)]]-16 \int\left[|x|^{2}|\varphi|^{2}+|x|^{2}|\psi|^{2}\right] d x-12 \int\left[|\varphi|^{2}+|\psi|^{2}\right] d x \\
= & 8\left[\frac{3}{2} L(\varphi, \psi)-\frac{1}{2} \int\left[|\nabla \varphi|^{2}+|\nabla \psi|^{2}+|x|^{2}|\varphi|^{2}+|x|^{2}|\psi|^{2}\right] d x\right]
\end{aligned}
$$




$$
\begin{aligned}
& -16 \int\left[|x|^{2}|\varphi|^{2}+|x|^{2}|\psi|^{2}\right] d x-12 \int\left[|\varphi|^{2}+|\psi|^{2}\right] d x \\
\leq & 8\left[\frac{3}{2} L(\varphi, \psi)-\frac{1}{2} \int\left[|\nabla \varphi|^{2}+|\nabla \psi|^{2}+|x|^{2}|\varphi|^{2}+|x|^{2}|\psi|^{2}\right] d x\right] \\
\leq & 8\left[\frac{3}{2} L(\varphi, \psi)-\frac{1}{2} \cdot \frac{3 \sqrt{2}}{4}\|Q\|_{2}^{2}\right] \\
= & 8\left[\frac{3}{2} L(\varphi, \psi)-\frac{3}{2} \cdot \frac{\sqrt{2}}{4}\|Q\|_{2}^{2}\right] \\
= & 12\left[L(\varphi, \psi)-\frac{\sqrt{2}}{4}\|Q\|_{2}^{2}\right] \\
= & 12\left[L\left(\varphi_{0}, \psi_{0}\right)-\frac{\sqrt{2}}{4}\|Q\|_{2}^{2}\right] \\
< & 0 .
\end{aligned}
$$

Then, from (4.4) and Lemma 2.3, using the argument of Glassey[10], it yields that the solutions $(\varphi, \psi)$ of the Cauchy problem (1.1) blow up in a finite time. The proof of Theorem 1.1 is complete.

Proof of Theorem 1.2

Assume $\left(\varphi_{0}, \psi_{0}\right) \in \Sigma \times \Sigma$. Let $(\varphi, \psi)$ be the solution of the Cauchy problem (1.1) corresponding the initial data $\left(\varphi_{0}, \psi_{0}\right)$. If $\left(\varphi_{0}, \psi_{0}\right)$ satisfy

$$
\begin{aligned}
\left\|\varphi_{0}\right\|_{\Sigma}^{2}+\left\|\psi_{0}\right\|_{\Sigma}^{2} & =\int\left[\left|\nabla \varphi_{0}\right|^{2}+\left|\nabla \psi_{0}\right|^{2}+|x|^{2}\left|\varphi_{0}\right|^{2}+|x|^{2}\left|\psi_{0}\right|^{2}+\left|\varphi_{0}\right|^{2}+\left|\psi_{0}\right|^{2}\right] d x \\
& <\frac{\sqrt{2}}{4}\|Q\|_{2}^{2},
\end{aligned}
$$

then it easily get $L\left(\varphi_{0}, \psi_{0}\right)<\frac{\sqrt{2}}{4}\|Q\|_{2}^{2}$.

Therefore, to prove Theorem 1.2, from Theorem 1.1, we only need to prove

$$
\int\left[\left|\nabla \varphi_{0}\right|^{2}+\left|\nabla \psi_{0}\right|^{2}+|x|^{2}\left|\varphi_{0}\right|^{2}+|x|^{2}\left|\psi_{0}\right|^{2}\right] d x<\frac{3 \sqrt{2}}{4}\|Q\|_{2}^{2} .
$$

From (4.5),

$$
\begin{aligned}
\int & {\left[\left|\nabla \varphi_{0}\right|^{2}+\left|\nabla \psi_{0}\right|^{2}+|x|^{2}\left|\varphi_{0}\right|^{2}+|x|^{2}\left|\psi_{0}\right|^{2}\right] d x } \\
& <\int\left[\left|\nabla \varphi_{0}\right|^{2}+\left|\nabla \psi_{0}\right|^{2}+|x|^{2}\left|\varphi_{0}\right|^{2}+|x|^{2}\left|\psi_{0}\right|^{2}+\left|\varphi_{0}\right|^{2}+\left|\psi_{0}\right|^{2}\right] d x \\
& <\frac{\sqrt{2}}{4}\|Q\|_{2}^{2} \\
& <\frac{3 \sqrt{2}}{4}\|Q\|_{2}^{2} .
\end{aligned}
$$

Therefore, (4.6) is true. The proof is complete. 


\section{Acknowledgements}

The authors would like to express their deep gratitude to Professor Jian Zhang for his helpful suggestions and advice. The project is supported by the National Science Foundation of China (Grant no. 10726033) the Scientific Research Found of Sichuan Provincial Education Department (Grant no. 2006A063) and the Scientific Research Found of Science and Technology Bureau of Sichuan Province (Grant no. 07JY029-012).

\section{References}

[1] P. BÉGOUT, Necessary conditions and sufficient conditions for global existence in the nonlinear Schrödinger equation, Adv. Math. Sci. Appl. 12(2) (2002), 817-827.

[2] H. BERESTYCKI and T. CAZENAVE, Instablilité des états stationnaires dans les équatons de Schrödinger et de Klein-Gordon non linéaires, $C$. $R$. Acad. Sci. Paris 293 (1981), 489-492.

[3] H. BERESTYCKI, T. GALLOUËT and O. KAVIAN, Équations de champs scalaires euclidiens nonlinéaires dans le plan, C. R. Acad. Sci. Paris. Serie I 297 (1983), 307-310.

[4] Y. B. BAND, M. TRIPPENBACH, J. P. BUKE and P. S. JULIENNE, Elasic scattering loss of atoms from colliding Bose-Einstein Condenste wave packets, Physical Review letters, 84(24) (2000), 5462-5465.

[5] T. BUSCH and J. R. ANGLIN, Dark-bright solitons in inhomogeneous Bose-Einstein Condensates, Physical Revies Letters 87(1) (2001), 01040110104014.

[6] T. CAZENAVE, An introduction to nonlinear Schrödinger equations, Textos de Metodos Matematicos, Vol.26, Rio de Janeiro, 1996.

[7] G. CHEN and J. ZHANG, Sharp threshold of global existence for nonlinear Gross-Pitaevskii equation in $R^{N}$, IMA Journal of Applied Mathematics, 71(2) (2006), 232-240.

[8] G. CHEN and J. ZHANG, Remarks on global existence for the supercritical nonlinear Schrödinger equation with a harmonic potential, Journal of Mathematical Analysis and Applications, 320(2) (2006), 591-598.

[9] G. CHEN and J. ZHANG, Threshold of global existence for the critical nonlinear Gross-Pitaevskii equation, Proceedings of the Edinburgh Mathematical Society, 50(1) (2007), 63-71. 
[10] R. T. GLASSEY, On the blowup of nonlinear Schrödinger equations, J. Math. Phys., 18(9) (1977), 1794-1797.

[11] J. GINIBRE and G. VELO, On a class of nonlinear Schrödinger equations, J. Funct. Anal., 32 (1979), 1-71.

[12] J. GINIBRE and G. VELO, The global Cauchy problem for the nonlinear Schrödinger equation, revisited, Ann. Inst. H. Poincaré Anal. Non Linéaire, 2 (1985), 309-327.

[13] E. A. KUZNETSOV, J. J. RASMUSSEN, K. RYPDAL and S. K. URITSYN, Sharper criteria for the wave collapse, Physica D, 87 (1995), 273-284.

[14] M. K. KWONG, Uniqueness of positive solutions of $\triangle u-u+u^{p}=0$ in $R^{N}$, Arch. Rat. Mech. Anal. 105(3) (1989), 243-266.

[15] Y. G. OH, Cauchy problem and Ehrenfest's law of nonlinear Schrödinger equations with potentials, Journal of Differential Equations, 81 (1989), $255-274$.

[16] T. OGAWA and Y. TSUTSUMI, Blow-up of $H^{1}$ solution for the nonlinear Schrödinger equation, J. Differential Equations, 92(2) (1991), 317-330.

[17] T. OGAWA and Y. TSUTSUMI, Blow-up of $H^{1}$ solution for the nonlinear Schrödinger equation with critic power nonlinearity, Proc. Amer. Math. Soc., 111(2) (1991), 487-496.

[18] T. OZAWA, Remarks on proofs of conservation laws for nonlinear Schrodinger equations, Calc. Var. Partial Differ. Equ., 25(3) (2006), 403-408.

[19] Q. H. PARK and J. H. EBERLY, Strong confinement and oscillations in two-component Bose-Einstein Condensates, Physical Review Letters, 85(20) (2000), 4195-4198

[20] W. A. STRAUSS, Existence of solitary waves in higher dimensions, Comm. Math. Phys., 55(2) (1977), 149-162.

[21] W. A. STRAUSS, Nonlinear wave equations, C.B.M.S. No.73, Amer. Math. Soc. Providence, Rl, 1989.

[22] R. TEMAM, Infinite dimensional dynamical systems in mechanics and physics, Springer-Verlag, New York, 1997.

[23] Y. TSUTSUMI and J. ZHANG, Instability of optical solitons for twowave interaction model in cubic nonlinear media, Advances in Mathematical Sciences and Applications, Gakkōtosho, Tokyo (1998), 691-713.

[24] M. I. WEINSTEIN, Nonlinear Schrödinger equations and sharp interpolations estimates, Commu. Math. Phys., 87 (1983), 567-576. 
[25] J. ZHANG, Sharp conditions of global existence for nonlinear Schrödinger and Klein-Gordon equations. Nonlinear Analysis: Theory Methods and Applications, 48 (2002), 191-207.

[26] J. ZHANG, Sharp threshold for global existence and blowup in nonlinear Schrödinger equation with harmonic potential, Communications in Partial Differential Equations, 30 (2005), 1429-1443.

Received 3 January 2007; accepted 17 May 2007; published Online First 10 January 2008

To access this journal online: http://www.birkhauser.ch 